\title{
An Optimization-Based Decision Support System for Strategic Planning in a Process Industry: The Case of a Pharmaceutical Company in India
}

\author{
Goutam Dutta * \\ Professor, Production and Quantitative Methods \\ Indian Institute of Management, Ahmedabad \\ Robert Fourer ** \\ Professor, Industrial Engineering and Management Sciences \\ Department of Industrial Engineering and Management Sciences \\ Northwestern University
}

\author{
Akhilesh Majumdar \\ Indian Institute of Technology, Delhi
}

Debabrata Dutta

TNS India Private Limited, Ahmedabad

* Professor, Production and Quantitative Methods, Indian Institute of Management, Vastrapur, Ahmedabad 380015

Phone: 91-79-26324828, Fax: 91-79-26324828

E-mail: goutam@iimahd.ernet.in

** Professor, Industrial Engineering and Management Sciences, Northwestern University, USA

Phone: 1-847-491-3151, Fax: 1-847-491-1719

E-mail: 4er@iems.northwestern.edu 


\begin{abstract}
We describe how a generic multi-period optimization-based decision support system (DSS) can be used for strategic planning in process industries. Built on five fundamental elements - materials, facilities, activities, storage areas and time periods - this DSS requires little direct knowledge of optimization techniques to be used effectively. It is user friendly and requires little knowledge of optimization. Results based on real data from a pharmaceuticals company in India demonstrate significant potential for improvements in revenues and profits.
\end{abstract}

This work has been supported by grants from the Research and Publication Committee of the Indian Institute of Management, Ahmedabad. 


\section{Introduction and Motivation}

The primary motivation for this work comes from previous work done by the authors (Dutta and Fourer, 2004, Dutta and Fourer, 2000), in which a generic optimization based decision support system was developed for strategic planning in process industries. This was then customized for an integrated steel plant in North America. The result was a potential increase of $16-17 \%$ in the bottom-line of the company. It was claimed that the same approach, being generic, could be applied to various other process industries. In this paper, we demonstrate the application of the same Decision Support System to a Pharmaceutical Company in India.

The applications of linear programming based techniques to a process industry (specially the steel industry) have been many. A series of publications (Dutta et al., 1994; Sinha et al., 1995, Dutta et al, 2000) report the conceptualization, development and implementation of a mixed integer linear programming model for optimal power distribution that took about 20 person years. This work resulted in a $58 \%$ increase in profitability (or a direct financial benefit of 73 million dollars) during the last six months of the fiscal year 1986-87, and accrued similar benefits in later years. However, in both of the above cases, the models were customized only for the steel industry.

The production system of a pharmaceutical industry is an intermittent one. It produces several types of drugs in varying quantities, which are routed through several machines and share a common set of resources. This leads to an inherently large number 
of constraints, making the determination of optimality go well beyond the scope of human comprehension and intuition and too complex to be attained manually. We therefore present this optimization based DSS which is aimed at providing strategic support to the pharmaceutical industry. In section 2, we give a brief account of previous attempts at applying OR/MS concepts in a pharmaceutical setup. In section 3, we discuss the basic approach of modeling in a process industry. The elements of the database required to define the mathematical model and the optimization steps are discussed in sections 4 and 5 respectively. In section 6 , we discuss the application of the model in a pharmaceutical company in India. The paper concludes by describing some of the experiments made on the model using real time data from the company and their results, illustrating the possible impact on the bottom-line in section 7. The mathematical formulation of our model is provided in the Appendix.

\section{Literature Review}

The literature pertaining to OR/MS applications in process industries is quite diverse and a comprehensive review is beyond the scope of this paper. However, a comprehensive survey of mathematical programming models in the steel industry (Dutta and Fourer, 2001) indicates that very little work has been done in the area of planning with multi-period linear programming models. Also, in contrast to the extensive literature on OR/MS applications in process industries, reported work in the context of DSS for pharmaceutical industries is fairly recent and quite sparse. 
Yang and Mou (1993) discuss the implementation of a LP based integrated DSS at the Dalian Dyestuff Plant, one of the largest of its kind in China. The DSS consisted of subsystems for production planning, accounting and finances, inventory, and information services. Operational results indicate that the system increased the annual profits by at least 4 million RMB in 1987 (about one million US dollars). However, not much was discussed about software implementation and actual results. Jager, Permoler and Robde (1989), present a DSS for time-phased planning of chemical components of active ingredients and intermediates in a pharmaceutical company in Netherlands. Artiba (1992) considers the case of a parallel multi-product and multi-machine production problem for the development of a knowledge based system in a pharmaceutical company. Two other papers (Vickery and Markland, 1986; Boykin R.F.,1985) focus specifically on optimizing production parameters like lot-sizes and other cost operating parameters.

Apart from the above, literature is also available on applications for network distribution and supply chain restructuring. Camm et al., (1997), implemented an integer programming based network optimization model to streamline work processes, drive out non-value-added costs, and eliminate duplication in order to restructure P\&G's supply chain in North America. Gupta et al., (2002), have reported the most recent work in this field. The authors constructed a DSS to help the distribution network of Pfizer/Warner Lambert. This improved the company's ability to take rapid, well-informed decisions in several areas of distribution and supply chain management ranging from individual customer deliveries to long term manufacturing location and technology issues. The 
development and validation of generic algorithm based facility layout is decribed in a work by Hamamoto et al. (1999). A complete different type of model is the queuing network model (Viswanadham and Narahari, 2001) for lead time compression for drug development.

As already discussed above, none of the available literature focuses on multi-period models. There is also a lack of sufficient literature that discusses software development and optimal results from implementation. Hence, this paper discusses the development of a multi-material, multi-facility, multi-activity and multi-period generic model, implementable across a spectrum of process industries. We conclude this section by noting that the current case of a pharmaceutical company, complemented by the previous success in integrated steel industries, is an effort to illustrate the same.

\section{Modeling a Process Industry}

In this section we describe our generic approach towards modeling a process industry. This approach is similar to the manner in which the authors have modeled a steel industry as mentioned before (Dutta and Fourer, 2004). We then go on to illustrate (in section 6) the application of this approach in a pharmaceutical company.

We characterize a process industry as a network consisting of several smaller manufacturing units/machines, through which several materials are routed and processed. 
Normally raw materials can only be bought, and finished products can only be sold. Intermediates can often neither be bought nor sold. Practically all material can be stored as inventory. At any time, we can set products bought, sold or inventoried to zero to indicate that no buying, selling or inventorying is possible. For each material the model also specifies a list of conversions to other materials. Each conversion has a yield and cost at any given time. This also takes care of recycling of materials.

The production of any product is much more difficult than a simple conversion. We define a collection of facilities at which transformation occurs. At any given time, each facility houses one or more activities, which use and produce material in certain proportions. We assume the production system to be continuously linear and hence we use linear models. The following information is provided for each unit activity at each facility at each time:

- The amount of each input required for an activity.

- The amount of each output resulting from an activity.

- The cost per unit of the activity.

- Upper and lower limits on the number of units of each activity.

- The number of units of activity that can be accommodated by one unit of the facility's capability. We call this the facility-activity ratio.

In defining an activity we have two different cases. In the first case, if there is more than one product being produced at any particular facility, the production of each product 
is modeled as a separate activity, since each activity produces a separate output. The units of an activity may be different from the units of the facility's capacity. For instance, the activity output might be measured in tons, but the facility's capacity might be specified in hours. In such situations the model specifies the facility-activity ratio as tons per hour. If two products are produced at the same facility with different production rates, we have two different activities and two different facility-activity ratios. In the other case, if a facility has essentially only one activity, both the activity and the facility capacity may be in the same units. The facility-activity ratio must be unity in this case.

Another important factor in the model is the definition of time. We take the time unit to be flexible from one day to one year. For long term capital budgeting and business planning, we would use a year, month or quarter as the unit of time, whereas for the short-term operational model, we use one week or one day as the unit of time. In the current case we have taken the unit of time to be one month as we attempt to provide long-term strategic support. With respect to strategic planning, the DSS is intended to help answer questions such as the following:

What is the optimal product mix and how does it compare to the current product $\operatorname{mix}$ ?

Which products should be chosen for addition to the existing products with the same facility.?

How does the nominal optimum vary from the discounted one? 
What is the effect of cost or price changes, of raw materials or finished products, on the product mix and overall profit?

In long-term planning or capital budgeting we need to calculate the discounted cash flow and thus the interest rate is an important factor.

\section{Model Formulation}

We optimize a generalized network-flow linear program based on five fundamental elements: materials, facilities, activities, storage areas and time periods. The details of the computer implementation are beyond the scope of this paper and are discussed in another paper (Dutta and Fourer, 2000). An appendix to this paper shows the model's complete formulation.

\subsection{Definitions}

The fundamental elements are defined as follows:

Times: These are the periods of the planning horizon and are represented by discrete numbers $1,2,3, \ldots, T$.

Materials: Any product in the manufacturing unit at any stage of production — input, intermediate, output — is regarded as a material. 
Facilities: A facility is a collection of machines which produce some materials from others. For example, a machine that mixes raw materials for tablets is a facility.

Activities: At any given time, each facility houses one or more activities, which use and produce material in certain proportions. In each activity at each time, we have one or more input materials being transformed into various output materials. Coating of tablet is an activity, the blister packing of the tablet, are examples of activities.

Storage areas: These are the warehouses where raw materials, intermediate products, and finished products can be stored.

The model is a generalized network-flow model that maximizes the contribution to profit (nominal or discounted) of a company, subject to the following categories of constraints for all time periods:

○ Material balances

- Facility capacities - optionally "soft" capacities that may be exceeded at some cost in outsourcing

- Storage area capacities

- Bounds - on material (bought, sold, inventoried), on facility inputs and outputs, and on activities 
Owing to the generality of the model, each material can potentially be bought, sold, or inventoried at each time period. Thus any material can be modeled as a raw material, intermediate, or finished product depending on the circumstances being considered.

\subsection{Assumptions}

The model we describe is general enough to accommodate facilities that are in series, in parallel, or in some more complex configuration. As previously remarked, each facility can have one or more activities.

There can be purchase, sale and storage of materials at the raw materials stage, at the finishing stage or at the intermediate processing stages. Moreover the purchase price of raw materials, the selling price of finished goods, and the inventory carrying costs may vary over time.

At any given time, one or more materials may be used as inputs or outputs at a facility. Generally more than one input material is used to produce one output. The relative proportions of inputs and outputs (the technological coefficients) of an activity remain the same in a given period. Technological coefficients may vary with time. 
The capacity of each facility and each storage area is finite. Since the facilities will have different patterns of preventive maintenance schedules, the capacities of the facilities will vary over time.

Costs and production amounts are considered to vary proportionately with activity levels. Thus essential features of the production-planning problem can be captured in a deterministic, linear optimization model.

\subsection{Implementation}

Our model is implemented within 4th Dimension (Adams and Becket, 1999), a relational database management system. Other database systems such as Access or Oracle could be used just as well.

Figure 1 shows the structure of the database as expressed within 4th Dimension. The five boxes labeled Materials, Facilities, Activities, Times, and Storage_Areas correspond to the five major elements or files of the database. Items within each box denote the file's data fields and subfiles, with the subfile entries distinguished by a light-shaded line that runs to the top of a separate box in which the subfile data fields are listed. The smaller, independent database structure in the upper middle of the diagram holds a generated linear program as described in the next subsection. 
Following 4th Dimension's notation, we use bracketed names to denote files and apostrophes to separate subfile and field names. Thus [Facilities] is the database file of facilities, [Facilities]Inputs is the subfile of facility inputs, and [Facilities]Inputs'InMin is a data field of the subfile. Further details can be found in the discussion of the one-period model in an earlier publication (Fourer, 1997). 


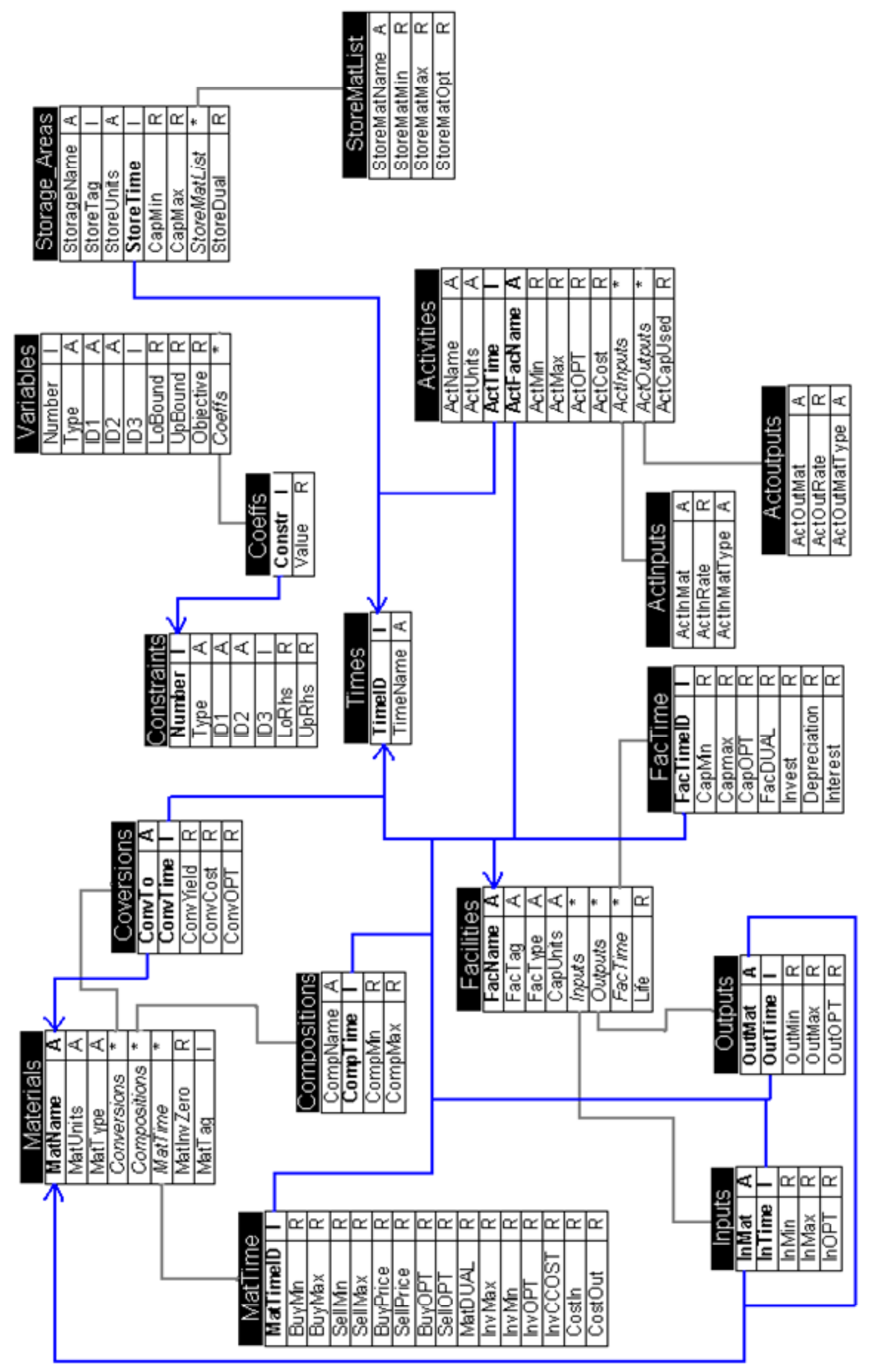

Figure 1: Database structure for the general planning model. 


\section{Optimization Steps}

Once the data of the five database files and their respective subfiles are entered, they are validated by a set of diagnostic tests which will be explained in section 6.3. This subsection describes how the subsequent optimization process is carried out. The principal steps (Figure 2) are as follows:

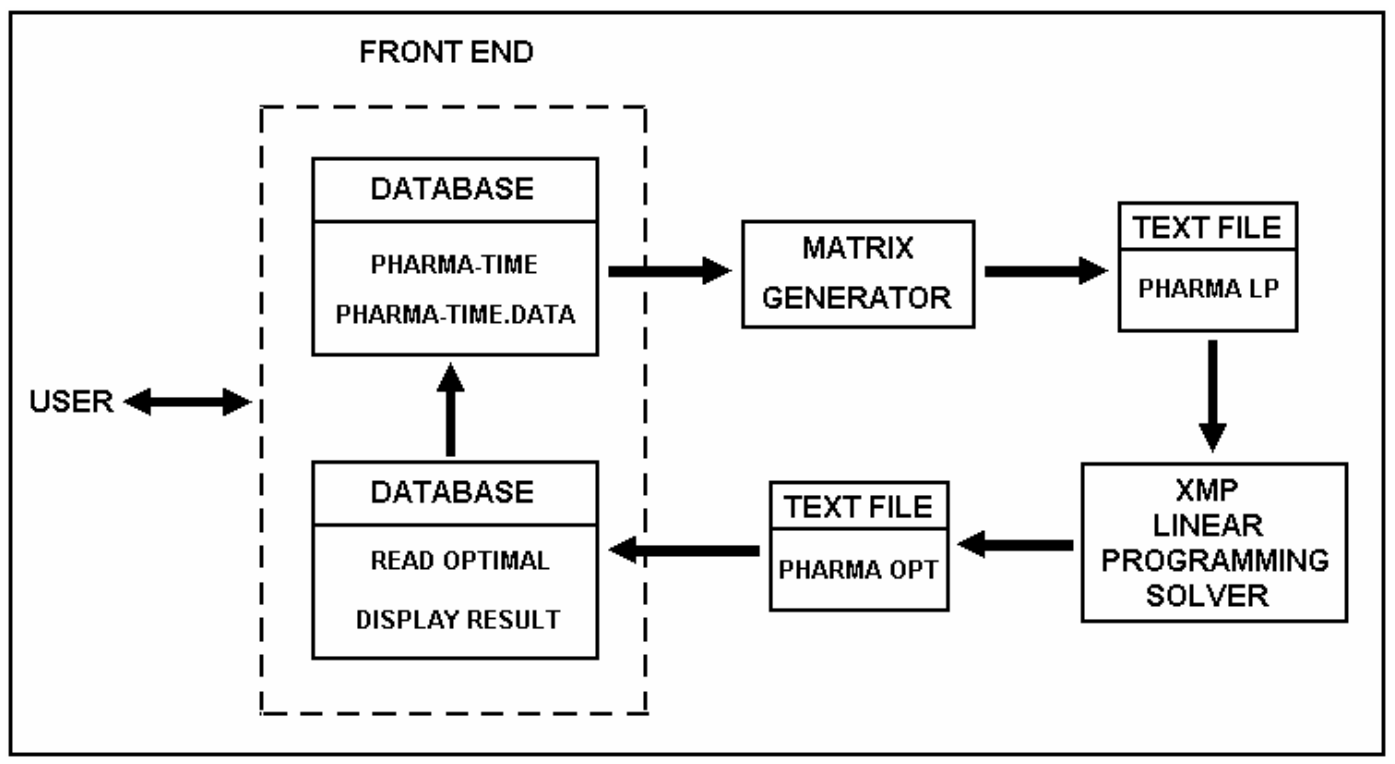

Figure 2: Optimization steps.

1. The data describing the production scenario at different time periods are collected and stored in the database.

2. The constraints associated with the linear program are generated. The constant terms of the constraint equations or inequalities, LoRHS and HiRHS, are extracted from the database and stored in the [Constraints] file. 
3. The variables of the associated linear program are determined, along with their coefficients in the constraints. Variables are stored in a separate [Variables] file and coefficients in its [Variables]Coeff subfile. This step gives the user a choice of discounted or undiscounted optimization. If the latter is chosen then it prompts for an interest rate, and all cost, price, and revenue data are converted to their discounted values in the objective function.

4. The [Constraints] and [Variables] files are scanned and all of the essential information about the linear program is written to an ordinary text file in a compact format. This text file is the input file to our solver.

5. A linear programming solver reads the text file—we used XMP (Martsen, 1981) - which solves the indicated linear program and then writes the optimal values of the variables to a second text file.

6. The second text file is read and the optimal values are placed in appropriate fields of the [Materials], [Facilities], [Activities], and [Storage_Areas] files and their subfiles.

To support these activities the database offers three modes of display. The Data mode is primarily for entering data describing the operations to be modeled. The Optimal mode also shows the fields for the optimal values, and hence is intended for examination 
of results. Finally, an Update mode allows small changes to be made to the data without a time-consuming re-generation of the [Constraints] and [Variables] files.

\section{Application to an Indian Pharmaceutical Company}

We have customized our DSS for strategic planning of an Indian pharmaceutical company. Spread over an area of 1,36,000 square meters, the plant is the largest of its kind at a single location. It consists of three main manufacturing units (Strategic Business Units - SBUs) functioning independently of each other. These are manufacturing units for liquid syrups, tablets and injections. The SBU for tablets produces seven types of tablets (prime materials) and the manufacturing process involves a total of 76 materials (including raw and intermediate materials) that are processed in 22 facilities housing a total of 51 activities. The corresponding figures for SBU for liquid syrups are 22 materials and 7 facilities housing 14 activities. For the injections SBU these figures correspond to 17 materials and 7 facilities housing 14 activities. Total revenue of this company is of the order of Rupees 8000 million (20 million USD). In this section we discuss how we set up an instance of our model using real data of the company. The specific questions we are interested in studying include:

What is the opportunity for increasing the profit of the company?

Which facilities have the most costly capacity limitations?

What are the processes that need the attention of the management? 
We also discuss the difficulties involved and indicate the impact that the DSS can make in improving the bottom line of a company.

\subsection{Conversion of the company's data to model data}

Due to reasons of confidentiality, the financial figures of the company, such as the purchase and sales prices of the materials and the costs of activities, were multiplied by a factor that was not disclosed. The exact input and output materials of each facility, yields for each activity, and capacities for different machines were supplied. At the same time, the actual names of products and facilities were replaced by code-names. The yield, capacity and facility activity ratios were the annual average values for the previous year. All of the results are based on the data presented to us in this disguised format.

Since the route of each product is different, the products at different stages were distinctly identified as different materials or different records in the [Materials] file. The facilities include mixing and binding units, drying machines, shifting machines, lubrication machines, compression and coating units and units for filtration, filling, leak testing, labeling, and blister and final packing. We decided to keep units of all materials in tons. The capacity of each facility was in hours and the capacity of each activity was also kept in tons. The facility activity ratio was thus expressed in tons per operating hour. The company did not supply a corresponding minimum number of operating hours, so [Facilities]FacTime'CapMin was set to zero. 
We were supplied with the data for a single period model of the production and financial parameters. Although the DSS was multi-period, this company decided to test the DSS for one year and supplied the data for one period. Therefore issues regarding inventories and discounting could not be tested. The final data set translated into 325, 78 and 92 variables and 244, 62 and 72 constraints corresponding to tablets, injections and liquid syrups respectively.

\subsection{Multiple processing in the same facility}

An important modeling issue was multiple processing at the same facility. As an example, in Figure 3 showing the processing of product PT1, the facility MT1 is used twice, once at the beginning and then again after MT7. Thus two stages of the same product are competing for the same facility capacity. This can be incorporated in the model, by defining two different activities at facility MT1 for the different processing stages. In the first stage activity, the input material is "PT1 at time 0 " and the output material is "PT1 after MT1-STAGE1". In the second stage activity, the input is "PT1 and RT3 after MT7" and the output is "PT1 after MT1-STAGE2." The interrelationship of these activities is handled implicitly in the overall solution of the LP. 


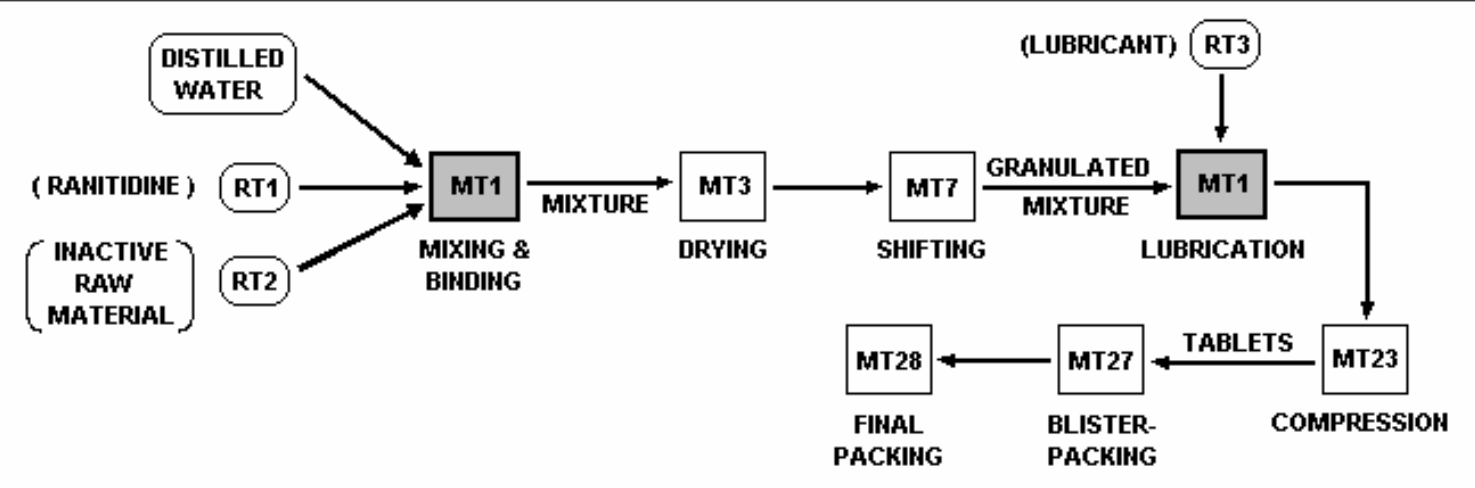

Figure 3: Process flow for product PT1, showing two different stages of processing at facility MT1.

\subsection{Verification}

To check that the model represents reality, contact points have been identified. These points are functions of the variables in the model and at the same time measurable quantities in real life. We consider the following figures and their respective units:

- Total production of the tablets unit (tons)

- Total production of the liquid syrups unit (tons)

- Total production of the injections unit (tons)

○ Total revenue (Rupees)

- Total cost of purchases (Rupees)

- Total cost of activities (Rupees)

- Total net profit (Rupees)

By comparing these quantities, we were able to verify that the results of the model were in line with the capabilities of the plant. 


\section{Experiments on the Model and Results}

We optimized with and without the company's actual production limits for the previous financial year. We also found the final products for which optimal values were at their upper limits ([Materials]MatTime'SellOPT= [Materials]MatTime'SellMax). We increased the upper bound by $5 \%$ for each final product. We re-ran the optimization model and noted the optimal results of the model. The same procedure was repeated twice more with an additional 5\% increase each time. The results of this study are shown in Table 1 for tablet manufacturing plants

Thus we considered the following four cases:

Case 0: With the company's upper and lower bounds

Case A: With the company's upper bounds increased by $5 \%$ over case 0

Case B: With the company's upper bounds increased by $5 \%$ over case A

Case C : With the company's upper bounds increased by $5 \%$ over case B 
(INSERT TABLE 1 HERE)

From the above table we recommend that the product PT-3 and PT-5 are not profitable for manufacturing and hence the company must stop producing these two products. Moreover, we also find that in the optimal solutions, the product PT-4 and PT-7 are at the upper limits. So for these products, the corresponding dual values are positive and marketing efforts need to be increased. For the other products, the company must maintain the existing production levels.

An analysis of Tables 3 and 4, which is fairly simple, indicates the following:

For Table 3, we find that there are only two product groups. Given this, the value of quantity in the optimal solution is at the upper limit. There was no capacity constraint in the machine. The company should try to increase marketing effort for each group of products. A similar explanation may be given for Table 4 .

( INSERT TABLE 2 HERE ) 
(INSERT TABLE 3 HERE)

(INSERT TABLE 4 HERE)

Our results confirm that a simple deterministic linear programming model can have significant impact in a pharmaceutical company. We can also draw comparisons with two previously mentioned studies (Dutta and Fourer, 2000 and Dutta et al., 1994) in the U.S. and India respectively. Both these works dealt with application of linear programming in integrated steel mills. The primary focus of the work in the U.S. was in the area of product-mix optimization, which resulted in potential improvements of $17-18 \%$ in the bottom-line of the company. The work in India dealt with optimal power distribution and resulted in a per ton profit improvement of 58\%. The focus of the work reported here is quite similar to the former one, showing that a generalized, linear programming based framework as applied here is very much applicable across a spectrum of process industries.

At this point we would like to discuss some of our experiences that we have observed with respect to the similarities and differences in modeling the same approach in two process industries.

1.In modeling an integrated steel plant, we used an completely integated model and one data set. While modeling Pharma Industry we found that the three SBU (Injection, tablet, 
Liquids) are not interrelated and we ran the optimization for three units separately and three data set.

2. The products in intermediate stages (including the in-process scrap generated) in steel plant has a market value, however, the similar products in pharmaceutical company do not have a market value.

3. In the steel industry facilities and processes are much more interlinked. In the steel plant, we have considered (Dutta and Fourer, 2004) the concept of recycling in the cold rolling mill, in the case of pharmaceuticals we did not have to apply that.

4. In the steel industry, some of the continuous plant cannot be switched off and runs on 24 hours 365 days basis. A pharma plant can be started and switched off everyday.

\section{Extension for Future Research}

We have studied a generic model for process industries that is multi-period, multifacility and multiple-activity, and that optimizes the nominal or discounted net profit of a company subject to the constraints of the industry. The problem can be visualized multiperiod single scenario or single period multi-scenario. We would like to extend it to multi-period, multi-scenario model. This requires that we define some of the data as stochastic with probability distributions. This will be a more difficult problem as the 
constraint generation and variable generation time will increase proportionately with the number of scenarios.

A second extension of the model will be studying the non-linearity of the model. Most of the industrial cost curves are non-linear or at best can be represented as having piecewise linear behavior.

A third extension will be to have multiple-objective linear programs and represent them in the database. This can be done by changing the model management system. For example, the current model can be changed to cost minimization, revenue maximization and maximization of marketable products or maximization of the utilization of marketable products (revenue or production). It is possible to have menu driven interfaces which allow for optimization over different objectives.

A fourth extension will be to attempt this DSS to another process industry. We have used the model for the steel and pharmaceutical industries. We need to test the model with real data from another process industry. In a developing country like India, we have a number of process industries. We need to test the DSS with other important process industries like the copper, aluminum, and fertilizers. A study relating to the aluminum industry is currently on and may be reported in forthcoming publications. 


\section{References}

Adams D. and Beckett D, Programming in $4^{\text {th }}$ Dimension, the Ultimate Guide, Automated Solutions Group: Huntington Beach, CA, U.S.A, 1999

Artiba C and Tahon C, 1992, Production Planning and Knowledge based System for Pharmaceuticals Manufacturing Company, European Journal of Operational Research, Volume 61, pp 18-29.

Boykin R. F. 1985, Optimizing Chemical Production at Monsanto, Interfaces, Volume 15, No. 1 (pp. 88-95)

Camm J.D. , Thomas C.E , Franz D.A. , James R.E., Sweeney J.D. Glenn W.W. , 1997, “ Blending OR/MS, Judgement and GIS: Restructuring P and G's Supply Chain, Interfaces, Volume 27, No. 1 pp 128-142.

Dutta G.; Sinha G. P; Roy P.N ; and Mitter N., 1994, “ A Linear Programming Model for Distribution of Electrical Energy in a Steel Plant," International Transactions in Operational Research, Volume 1, No 1. pp. 17-29.

Dutta G. and Fourer R., 2001 "A Survey of Linear Programming Applications in an Integrated Steel Plant," Manufacturing and Service Operations Management, Vol. 3., No.4, Fall 2001, pp. 387-400.

Dutta G. and Fourer R., 2000, "Database Structure for a Class of Multi-period Mathematical Programming Models", http://users.iems.northwestern.edu/ 4er/WRITINGS/index.html\#datamultil0 $\underline{5}$

Dutta G. and Fourer R.,2004, “An Optimization-Based Decision Support System for Strategic and Operational Planning in Process Industries", Optimization and Engineering, Volume 5, No. 3 (2004) pp. 295-314

Fourer R. 1997, Database Structure for Mathematical Programming Models, Decision Support Systems. Volume 20, pp. 317-344.

Gupta V., Peters E., Miller T., Kelvin B., 2002 Implementing a Distribution Network Decision Support System at Pfizer/ Warner Lambert, Interfaces, Volume 32, No. 4, pp. 28-25.

Hamamoto, S. Yih, Y. and Salvendy, G., 1999, Development And Validation Of Genetic Algorithm-Based Facility Layout - A Case Study In The Pharmaceutial Industry, International Journal of Production Research, Vol.37. No.4, pp. 749-768. 
Jager K, Permoller W, and Robde M, 1989, “ A Decision Support System for Planning Chemical Production of Active Ingredients in a Pharmaceuticals Company", Engineering Cost and Production Economics, The Netherlands, Volume 1 No. 4, pp. 377-387

Marsten R.E., 1981. The Design Of XMP Linear Programming Library, ACM Transactions on Mathematical Software. Volume 7, pp. 481-497.

Sinha G. P., Chandrashekaran B.S., Mitter N., Dutta G., Singh S.B., Roy P.N., and Roy Choudhary A., 1995, "Strategic and Operations Management with Optimization at Tata Steel" Interfaces, Volume 25, No. 1

Vickery S.K. and Markland E.R. , 1986, “ Multi-stage Lot Sizing in Serial Production System” International Journal of Production Research ”, Vol. 24 no. 3, pp 517-534

Viswanadham, N. and Narahari, Y., 2001, Queueing Network Modelling and Lead Time Compression of Pharmaceutical Drug Development, International Journal of Production Research, Vol.: 39 No. 2, pp. 395-412

Yang D. and Mou W., 1993"An Integrated Decision Support System for Chinese Chemical Plant", Interfaces, Vol. 27. No. 1, pp128-142. 


\section{Appendix}

\section{Model Formulation}

We first define the data, in five parts: times, materials, facilities, activities, and storage-areas. The notation for the decision variables is then presented. Finally the objective and constraints are described, in both words and formulae.

All quantities of materials are taken to be in the same units, such as kilograms.

\section{Time data}

$T=\{1, \ldots \ldots, T\}$ is the set of time periods in the planning horizon, indexed by $\mathrm{t}$ $\rho$ is the interest rate per period, taken as zero if there is no discounting

\section{Materials data}

$M$ is the set of all materials

$l_{j t}^{b u y}=$ lower limit on purchases of material $j$, for each $j \in M$ and $t \in T$

$u_{j t}^{b u y}=$ upper limit on purchases of material $j$, for each $j \in M$ and $t \in T$

$\mathcal{C}_{j t}^{b u y}=$ cost per unit of material $j$ purchased, for each $j \in M$ and $t \in T$

$l_{j t}^{\text {sell }}=$ lower limit on sales of material $j$, for each $j \in M$ and $t \in T$

$u_{j t}^{\text {sell }}=$ upper limit on sales of material $j$, for each $j \in M$ and $t \in T$

$\mathcal{C}_{j t}^{\text {sell }}=$ revenue per unit of material $j$, for each $j \in M$ and $t \in T$

$l_{j t}^{i n v}=$ lower limit on inventory of material $j$, for each $j \in M$ and $t \in T$

$\mathcal{U}_{j t}^{i n v}=$ upper limit on inventory of material $j$, for each $j \in M$ and $t \in T$

$v_{j 0}^{i n v}=$ initial inventory of material $j$, for each $j \in M$

$\mathcal{C}_{j t}^{i n v}=$ holding cost per unit of material $j$, for each $j \in M$ and $t \in T$

$M^{\text {conv }} \subseteq\left\{j \in M, j^{\prime} \in M: j \neq j^{\prime}\right\}$ is the set of conversions: 


$$
\begin{aligned}
& \left(j, j^{\prime}\right) \in M^{\text {conv }} \text { means that material } j \text { can be converted to material } j^{\prime} \\
\alpha_{j j^{\prime} t}^{c o n v}= & \text { number of units of material } j^{\prime} \text { that result from converting one unit of material } j, \\
& \text { for each }\left(j, j^{\prime}\right) \in M^{c o n v}, t \in T \\
\mathcal{C}_{j j^{\prime} t}^{c o n v}= & \text { cost per unit of material } j \text { of the conversion from } j \text { to } j^{\prime}, \text { for each }\left(j, j^{\prime}\right) \in M^{c o n v}, \\
& t \in T
\end{aligned}
$$

\section{Facilities data}

$\boldsymbol{F}$ is the set of facilities

$l_{i t}^{\text {cap }}=$ the minimum amount of the capacity of facility $i$ that must be used, for each $i \in F$ and $t \in T$

$u_{i t}^{c a p}=$ the capacity of facility $i$, for each $i \in F$ and $t \in T$

$\mathcal{C}_{i t}^{c a p}=$ the cost of vendoring (outsourcing) a unit of capacity at facility $i$, for each $i \in F$ and $t \in T$

$F^{\text {in }} \subseteq F x M$ is the set of facility inputs:

$(i, j) \in F^{\text {in }}$ means that material $j$ is used as an input at facility $i$

$l_{i j t}^{i n}=$ the minimum amount of material $j$ that must be used as input to facility $i$, for each $(i, j) \in F^{i n}, t \in T$

$u_{i j t}^{i n}=$ the maximum amount of material $j$ that must be used as input to facility $i$, for each $(i, j) \in F^{i n}, t \in T$

$F^{\text {out }} \subseteq F x M$ is the set of facility outputs:

$(i, j) \in F^{\text {out }}$ means that material $j$ is produced as an output at facility $i$

$l_{i j t}^{\text {out }}=$ the minimum amount of material $j$ that must be produced as output at facility $i$, for each $(i, j) \in F^{\text {out }}, t \in T$

$\boldsymbol{U}_{i j t}^{\text {out }}=$ the maximum amount of material $j$ that must be produced as output at facility $i$, for each $(i, j) \in F^{\text {out }}, t \in T$

\section{Activities data}

$F^{a c t} \subseteq\{(i, k): i \in F\}$ is the set of activities:

$(i, k) \in F^{a c t}$ means that $k$ is an activity available at facility $i$ 
$l_{i k t}^{a c t}=$ the minimum number of units of activity $k$ that may be run at facility $i$, for each $(i, k) \in F^{a c t}, t \in T$

$\mathcal{U}_{i k t}^{a c t}=$ the maximum number of units of activity $k$ that may be run at facility $i$, for each $(i, k) \in F^{a c t}, t \in T$

$\mathcal{C}_{i k t}^{a c t}=$ the cost per unit of running activity $k$ at facility $i$, for each $(i, k) \in F^{a c t}, t \in T$

$r_{i k t}^{a c t}=$ the number of units of activity that can be accommodated in one unit of capacity of facility $i$, for each $(i, k) \in F^{a c t}, t \in T$

$A^{i n} \subseteq\left\{(i, j, k, t):(i, j) \in F^{i n}(i, k) \in F^{a c t}, t \in T\right\}$ is the set of activity inputs:

$(i, j, k, t) \in A^{i n}$ means that input material $j$ is used by activity $k$ at facility $i$ during time period $t$

$\alpha_{i j k t}^{i n}=$ units of input material $j$ required by one unit of activity $k$ at facility $i$ in time period $t$, for each $(i, j, k, t) \in A^{i n}$

$A^{\text {out }} \subseteq\left\{(i, j, k, t):(i, j) \in F^{\text {out }}(i, k) \in F^{a c t}, t \in T\right\}$ is the set of activity outputs:

$(i, j, k, t) \in A^{\text {out }}$ means that output material $j$ is produced by activity $k$ at facility $i$ during time period $t$

$\alpha_{i j k t}^{\text {out }}=$ units of output material $j$ produced by one unit of activity $k$ at facility $i$ in time period $t$, for each $(i, j, k, t) \in A^{\text {out }}$

\section{Storage-areas data}

$S$ is the set of storage areas

$l_{s t}^{\text {stor }}=$ lower limit on total material in storage area $\mathrm{s}$, for each $\mathrm{s} \in S, t \in T$

$u_{s t}^{\text {stor }}=$ upper limit on total material in storage area $\mathrm{s}$, for each $\mathrm{s} \in S, t \in T$

\section{Variables}

$x_{j t}^{b u y} \quad=$ units of material $j$ bought, for each $j \in M, t \in T$

$x_{j t}^{\text {sell }}=$ units of material $j$ sold, for each $j \in M, t \in T$

$x_{j s t}^{\text {stor }}=$ units of material $j$ in storage area $s$, for each $j \in M, \mathrm{~s} \in S, t \in T$

$x_{j t}^{i n v}=$ total units of material $j$ in inventory (storage), for each $j \in M, t \in T$

$x_{j 0}^{i n v}=$ initial inventory of material $j$, for each $j \in M$

$x_{j j^{\prime} t}^{c o n v}=$ units of material $j$ converted to material $j^{\prime}$, for each $\left(j, j^{\prime}\right) \in M^{\text {conv }}, t \in T$ 
$x_{i j t}^{i n} \quad=$ units of material $j$ used as input by facility $i$, for each $(i, j) \in F^{i n}, t \in T$

$x_{i j t}^{\text {out }}=$ units of material $j$ produced as output by facility $i$, for each $(i, j) \in F^{\text {out }}, t \in T$

$x_{i k t}^{a c t}=$ units of activity $k$ operated at facility $i$, for each $(i, k) \in F^{a c t}, t \in T$

$x_{i t}^{c a p}=$ units of capacity vendored at facility $i$, for each $\mathrm{i} \in F, t \in T$

\section{Objective}

Maximize the sum over all time periods of revenues from sales less costs of purchasing, holding inventories, converting, operating activities at facilities and vendoring:

$\sum_{t \in T}(1+\rho)^{-t} Z(t)$

where,

$$
\begin{aligned}
\mathrm{Z}(\mathrm{t})= & \sum_{j \in M} \mathcal{C}_{j t}^{\text {sell }} x_{j t}^{\text {sell }}-\sum_{j \in M} \mathcal{C}_{j t}^{b u y} x_{j t}^{b u y}-\sum_{j \in M} \mathcal{C}_{j t}^{i n v} x_{j t}^{i n v}-\sum_{\left(j, j^{\prime}\right) \in M^{c o n v}} \mathcal{C}_{j j^{\prime} t}^{c o n v} x_{j j^{\prime} t}^{c o n v}-\sum_{(i, k) \in F^{a c t}} \mathcal{C}_{i k t}^{a c t} x_{i k t}^{a c t} \\
& -\sum_{i \in F} \mathcal{C}_{i t}^{c a p} x_{i t}^{c a p}
\end{aligned}
$$

\section{Constraints}

For each $j \in M, \mathrm{r} \in R$ and $t \in T$, the amount of material $j$ made available by purchases, production, conversions and beginning inventory must equal the amount used for sales, production, conversions and ending inventory:

$$
\begin{aligned}
& x_{j t}^{\text {sell }}+\sum_{(i, j) \in F^{o u t}} x_{i j t}^{o u t}+\sum_{\left(j^{\prime}, j\right) \in M^{\text {conv }}} \alpha_{j^{\prime} j t}^{\text {conv }} x_{j^{\prime} j t}^{c o n v}+x_{j t-1}^{i n v}=x_{j t}^{b u y}+\sum_{(i, j) \in F^{i n}} x_{i j t}^{i n}+ \\
& \sum_{\left(j, j^{\prime}\right) \in M^{\text {conv }}} x_{j j^{\prime} t}^{\text {conv }}+x_{j t}^{i n v}
\end{aligned}
$$

For each $(i, j) \in F^{i n}$ and $t \in T$, the amount of input $j$ used at facility $i$ must equal the total consumption by all the activities at facility $i$ :

$$
x_{i j t}^{i n}=\sum_{(i, j, k, t) \in A^{i n}} \alpha_{i j k t}^{i n} x_{i k t}^{a c t}
$$

For each $(i, j) \in F^{\text {out }}$ and $\mathrm{t} \in T$, the amount of output $j$ produced at facility $i$ must equal the total production by all the activities at facility $i$ :

$$
x_{i j t}^{\text {out }}=\sum_{(i, j, k, t) \in A^{o u t}} \alpha_{i j k t}^{o u t} x_{i k t}^{a c t}
$$


For each $\mathrm{i} \in F$ and $t \in T$, the capacity used by all activities at facility $i$ must be within the range given by the lower limit and the upper limit plus the amount of capacity vendored:

$l_{i t}^{c a p} \leq \sum_{(i, k) \in F^{a c t}} x_{i k t}^{a c t} / r_{i k t}^{a c t} \leq \mathcal{U}_{i t}^{c a p}+x_{i t}^{c a p}$

For each $j \in M$, the amount of material inventoried in the plant before the first time period is defined to equal the specified initial inventory:

$x_{j 0}^{i n v}=v_{j 0}$

For each $j \in M$ and $t \in T$, the total amount of material $j$ inventoried is defined as the sum of the inventories over all storage areas:

$\sum_{s \in S} x_{j s t}^{s t o r}=x_{j t}^{i n v}$

For each $\mathrm{s} \in S$ and $t \in T$, the total of all materials inventoried in storage area $s$ must be within the specified limits:

$l_{s t}^{s t o r} \leq \sum_{j \in M} \mathcal{X}_{j s t}^{\text {stor }} \leq \mathcal{U}_{s t}^{\text {stor }}$

All variables must lie within the relevant limits defined by the data:

$l_{j t}^{b u y} \leq x_{j t}^{b u y} \leq u_{j t}^{b u y}, \quad$ for each $j \in M$ and $t \in T$

$l_{j t}^{\text {sell }} \leq x_{j t}^{\text {sell }} \leq u_{j t}^{\text {sell }}, \quad$ for each $j \in M$ and $t \in T$

$l_{j t}^{i n v} \leq x_{j t}^{i n v} \leq u_{j t}^{i n v}, \quad$ for each $j \in M$ and $t \in T$

$0 \leq x_{j j^{\prime} t}^{c o n v}$,

for each $\left(j, j^{\prime}\right) \in M^{\text {conv }}$ and $t \in T$

$0 \leq x_{i t}^{c a p}$

for each $\mathrm{i} \in F$ and $t \in T$

$0 \leq x_{j s t}^{\text {stor }}$,

for each $\mathrm{s} \in S, j \in M$ and $t \in T$

$l_{i j}^{i n} \leq x_{i j}^{i n} \leq u_{i j}^{i n}$,

for each $(i, j) \in F^{i n}$ and $t \in T$

$l_{i j}^{\text {out }} \leq x_{i j}^{\text {out }} \leq u_{i j}^{\text {out }}$,

for each $(i, j) \in F^{\text {out }}$ and $t \in T$

$l_{i k}^{a c t} \leq x_{i k}^{a c t} \leq u_{i k}^{a c t}$,

for each $(i, j) \in F^{a c t}$ and $t \in T$ 
Table 1: Results of Optimization Experiment in Tablet Units

\begin{tabular}{|l|r|r|r|r|}
\hline Material & Case 0 & Case A & Case B & Case C \\
\hline PT-1 & 2171.6 & 2171.6 & 2171.6 & 2171.6 \\
\hline PT-2 & 131.04 & 131.04 & 131.04 & 131.04 \\
\hline PT-3 & 0 & 0 & 0 & 0 \\
\hline PT-4 & $\mathbf{1 2 7 . 4}$ & $\mathbf{1 3 3 . 2}$ & $\mathbf{1 4 0 . 2 . 4}$ & $\mathbf{1 4 7 . 2}$ \\
\hline PT-5 & 1987.4 & 1987.4 & 1987.4 & 1987.4 \\
\hline PT-6 & $\mathbf{5 8 8 0}$ & $\mathbf{6 1 7 4}$ & $\mathbf{6 4 8 2}$ & $\mathbf{6 8 0 6}$ \\
\hline PT-7 & 10297 & 10597 & 10913 & 11243 \\
\hline Total & & & & 0 \\
\hline
\end{tabular}


Table 2 : Optimization Results:Tablets

\begin{tabular}{|c|c|c|c|c|c|c|}
\hline \multirow{5}{*}{$\frac{\sqrt{-1}}{\frac{9}{6}}$} & Variables & case 0 & case $A$ & case B & case $\mathrm{C}$ & Change \\
\hline & Revenue & $34,549,486$ & $34,947,314$ & $35,365,064$ & $35,803,658$ & 3.50 \\
\hline & Cost of purchase & $6,290,272$ & $6,417,152$ & $6,550,379$ & $6,690,263$ & 5.97 \\
\hline & Cost of activities & $1,501,725$ & $1,571,925$ & $1,645,659$ & $1,723,079$ & 12.84 \\
\hline & Net Profit & $26,757,489$ & $26,958,237$ & $27,169,026$ & $27,390,316$ & 2.31 \\
\hline
\end{tabular}

Table 3 : Optimization results: Injections

\begin{tabular}{|c|c|c|c|c|c|c|}
\hline \multirow{5}{*}{$\begin{array}{l}z \\
\text { 음 } \\
\text { U. } \\
\text { ㅁ } \\
\underline{z}\end{array}$} & Variables & case 0 & case $A$ & case $B$ & case $\mathrm{C}$ & Change \\
\hline & Revenue & $8,902,200$ & $9,347,310$ & $9,814,676$ & $10,305,178$ & 15.76 \\
\hline & Cost of purchase & 84,273 & 88,514 & 92,968 & 97,556 & 15.76 \\
\hline & Cost of activities & 9,339 & 9,806 & 10,760 & 10,880 & 15.76 \\
\hline & Net Profit & $8,808,588$ & $9,248,989$ & $9,710,948$ & $10,196,742$ & 15.76 \\
\hline
\end{tabular}

Table 4 : Optimization results: Liquid

\begin{tabular}{|l|l|c|c|c|c|c|}
\hline \multirow{4}{*}{} & Variables & case 0 & case A & case B & case C & Change \\
\cline { 2 - 7 } & Revenue & $755,200,585$ & $792,960,616$ & $832,608,650$ & $874,239,078$ & 15.76 \\
\cline { 2 - 7 } & Cost of purchase & $35,154,506$ & $36,917,481$ & $38,763,355$ & $40,695,735$ & 15.76 \\
\cline { 2 - 7 } & Cost of activities & 329,367 & 345,835 & 363,127 & 381,284 & 15.76 \\
\cline { 2 - 7 } & Net Profit & $719,711,712$ & $755,697,300$ & $793,482,167$ & $833,162,059$ & 15.76 \\
\hline
\end{tabular}


Author Details

Goutam Dutta is a professor of Production and Quantitative Methods Area at Indian Institute of Management, Ahmedabad

Robert Fourer is a professor of Industrial Engineering and Management Sciences at Northwestern University

Akhilesh Majumdar is a former student of Indian Institute of Technology, Delhi

Debrabata Dutta is working in TNS India, a market research consultancy firm 